der Christoph Merian Stiftung an der „Grün 99“ in Weil am Rhein an acht Computerstationen tun. Und wer bereits jetzt den Gang in die Buchhandlung antritt, der kann sich auf eine ausgedehnte spannende Reise durch die Zeit, Natur und Kultur der Region Basel freuen.

Systemanforderungen: 4x-CD-Laufwerk, Monitor für 16-Bit-Farbtiefe bei $800 \times 600$ Pixeln, 24 MB RAM, Windows: ab 486-Prozessor (empfohlen: Pentium), Windows 95 oder 98, NT ab Version 3.5.1, SoundBlasterkompatible Soundkarte, Macintosh ab 68040 Prozessor (empfohlen: Power-Macintosh), Mac OS 7.1 oder höher.

Martin Rickenbacher, Basel

\section{Hinweise auf Neverscheinungen}

Das Bundesamt für Kartographie und Geodäsie bietet jetzt sei- ne Amtlichen Topographischen Karten der Maßstäbe 1:200000, $1: 500000$ und $1: 1$ Mio. flächendeckend und blattschnittfrei für das Gebiet der Bundesrepublik Deutschland auf der CD-ROM „Top200“ an. Auf der CD-ROM befinden sich außerdem ein Ortsnamenverzeichnis mit ca. 46000 Einträgen sowie die Verwaltungsgrenzen.

Die Systemvoraussetzungen sind: IBM-kompatibler PC ab 486, 8 MB RAM, Windows 3.1, Win95 oder WinNT, Graphikkarte mit 256 Farben. Interessenten erhalten die CD-ROM „Top200“ zum Preis von DM 78,00 zuzüglich Versandkosten beim Bundesamt für Kartographie und Geodäsie, Abt. GI-K1.1, Richard-StraussAllee 11, 60598 Frankfurt am Main, Fax: 069-631 4905 26; Internet: http://www.ifag.de; oder im Buchhandel unter ISBN-Nummer 3-88648-055-0.
Bei der Wissenschaftlichen Buchgesellschaft haben kürzlich Th. Ott und P. Tiedemann das Buch „Internet für Geographen“ veröffentlicht. Wie der Untertitel ausweist, handelt es sich um eine „praxisorientierte Einführung “ (Redaktionsschluß Januar 1999), die durchaus auch das Interesse der Kartographie verdient. Dies gilt nicht nur für Teil I, der in das Internet einführt, oder Teil III, der Fragen des Publizierens im Internet behandelt, sondern auch für Teil II, in dem wichtige Anschriften vorgestellt werden. Hier finden auch Kartographen interessante Anschriften, so etwa in Kapitel 10 (Kartographie und GIS). - Ott, Thomas und Paul Tiedemann: Internet für Geographen. Eine praxisorientierte Einführung. Darmstadt: Wissenschaftliche Buchgesellschaft 1999; XII + 143 Seiten, 47 SW-Abbildungen. Bestellnummer: 14162-8.

\title{
Veranstaltungen 1999
}

14. -21. 8. 11. Generalversammlung der Internationalen Kartographischen Vereinigung (IKV/ICA/ACI) und 19. Internationaler Kartographischer Kongreß in Ottawa, Kanada, organisiert durch: The Canadian Institute of Geomatics/Association Canadienne des Sciences Géomatiques (s. auch KN 4/98, S. 166). Anmeldung bei: ICA Ottawa 1999, 615 Booth Street, Room 500, Ottawa, Ontario K1A OE9, Canada; Tel.: (613) 992-9999; Fax: (6 13) 995-8737; E-Mail: ica1999@ccrs.nrcan.gc.ca; Internet: www.ccrs.nrcan.gc.ca/ica1999/

20. -23. 9. „Der Einsatz von (GIS) in der Planungspraxis“ an der Hochschule Vechta, Abteilung GIS, und vom

27.9.- 1. 10. „GIS Kombi-Kurs“ an der Hochschule Vechta, Abteilung GIS. Anmeldung bei: Hochschule Vechta/ISPA, z. Hd. Frau Klein, Postfach 1553, D-49364 Vechta; Tel.: 04441-15344; FAX: 0444115445; E-Mail: iklein@ispa.uni-vechta.de

20. -24. 9. 47. Photogrammetrische Woche mit vorangestelltem Tutorium (s. KN 2/99, S. 73).

22. -25. 9. digiMedia 99 - Internationale Messe und Kongreß für Publishing, Marketing und Kommunikation. Messe Düsseldorf, Postfach 101006, D-40001 Düsseldorf, Tel.: +49(0)2 11/4560-01; Fax: $+49(0) 211 / 4560-548$

27. -28. 9. „Dresdener Sommerschule für Kartographie“ (s. S. 170).

6. -8.10 . 19. Wissenschaftlich-Technische Jahrestagung der Deutschen Gesellschaft für Photogrammetrie und Fernerkundung e.V. (DGPF) „Neue Sensoren - neue Anwendungen“ in Essen, Universität Gesamthochschule. Aktuelle Informationen über http://www.dgpf.de.

\section{Veranstaltungen 2000}

10. -12. 5. Symposium Praktische Kartographie 2000 „Neue Wege für die Kartographie?“ (s. in diesem Heft S. 173).

18. -31. 5. drupa 2000 „print media messe“. Pressereferat drupa 2000, Monika Kissing, Postfach 101006 , D-40001 Düsseldorf; Telefon: +49(0)2 11/4560-543, Fax: +49(0)2 11/45 60-5 48, E-Mail: KissingM@nowea.de

14. -16. 9. 10. Kartographiehistorisches Colloquium und Sitzung der Arbeitsgruppe D-A-CH in Bonn (s. KN $1 / 99$, S. 25)

11. -14.10. 49. Deutscher Kartographentag und Intergeo Berlin: „Rauminformationen für das 21. Jh.“; 50 Jahre DGfK. 